\title{
La Unión Europea en la Gobernanza Global*
}

\author{
The European Union in Global Governance
}

\author{
Nicolás Mariscal \\ Profesor emérito de la Universidad de Deusto, Profesor Jean Monnet emérito \\ nicolas.mariscal@deusto.es
}

doi: http://dx.doi.org/10.18543/ced-56-2017pp181-204

\begin{abstract}
Sumario: I. Introducción.-II. Dos procesos del surgimiento de la gobernanza global. 1. Los poderes emergentes. 2. La globalización.III. La Unión Europea en la gobernanza global.--IV. La UE un actor global. 1. Presencia y «actoridad»(actorness). 2. Estrategias europeas globales.-V. La Unión Europea como un actor diplomático.- VI. La percepción de la UE por otros actores de la gobernanza global.-VII. A modo de conclusión.
\end{abstract}

Resumen: Dos grandes procesos de finales del siglo XX y comienzos del XXI han condicionado el surgimiento de la gobernanza global: los poderes emergentes y la globalización. La gobernanza global es un proceso y una estrategia en que una pluralidad de actores públicos y privados de diversos niveles interactúan y cooperan sin un Gobierno común, para manejar problemas específicos desnacionalizados o proveer bienes públicos transnacionales. Es una casa en medio del camino entre la anarquía internacional y un Estado mundial. La Unión Europea trata de ser un actor global y para ello ha elaborado en el presente siglo diversas estrategias europeas globales. Aquí se presenta la última de 2016 con cinco grandes prioridades. Como actor diplomático la UE es percibida y valorada diferentemente por otros que con ella interactúan en la gobernanza global.

Palabras clave: integración europea, gobernanza global, teorías, estrategias, «actoría»y «actoridad».

Abstract: Two major processes of the late twentieth and early twenty-first have conditioned the emergence of global governance: emerging powers and globalization. Global governance is a process and strategy in which a plurality of public and private actors of different levels interact and cooperate without a common government, to handle specific denationalized problems or to provide transnational public goods. It is a house in the middle of the road between international anarchy and a world state. The European Union tries to be a global player and for this purpose has developed several global European strategies in the

* Recibido el 15 de noviembre de 2016, aceptado el 22 de febrero de 2017. 
present century. Here is the last of 2016 with five major priorities. As a diplomatic actor, the EU is perceived and valued differently by others who interact with it in global governance.

Keywords: European integration, global governance, theories, strategies, «actorship» and «actorness».

\section{Introducción}

La globalización es un macrofenómeno societal de finales del siglo XX y comienzos del XXI, objeto de numerosas y ricas conceptualizaciones: estructuración del mundo como un todo; profundo cambio ontológico; sociedad de la información y redes globales; compresión espacio-temporal transformadora; crítica de ésta desde la comprensión histórico-sociológica del poder social del capitalismo; expresión de un todo global; diferentes y cambiantes globalizaciones. ${ }^{1}$

En los dos primeros decenios del presente siglo XXI se ha correlacionado - sin entrar ahora en la complejísima cuestión de las causalidades- con una fuerte conflictividad política mundial, que en el cambio del milenio por un momento soñamos superada: las agresiones de septiembre de 2001 a Estados Unidos y las consiguientes guerras de Afganistán e Irak, la crisis financiera y económica desde 2008 y sus consecuencias de desempleo y desigualdad, las primaveras árabes tornadas violencia y penosas emigraciones, el ascenso de China, el triste renquear de la Unión Europea, Crimea, Ucrania, Siria, el regreso de Rusia como potencia mundial, los ubicuos esfuerzos de renacionalización, el Brexit y el huracán Trump... El globo se vuelve a mostrar peligroso y de alto riesgo.

En esta situación mundial la gobernanza global adquiere una crucial relevancia como búsqueda de salida a los conflictos que amenazan a la humanidad. Europa quiere y todavía podría jugar un rol decisivo en esa búsqueda y realización, si...

El presente artículo tiene carácter introductorio y pretende proporcionar una panorámica teórica general, una visión holística de la Unión Europea como un actor de la gobernanza global. Comienza apuntando a dos procesos - los poderes emergentes y la globalización - que han contribuido al surgimiento y desarrollo reciente de la gobernanza global (II), para cen-

1 Nicolás Mariscal, «Globalización-Unión Europea-Globalización», Cuadernos Europeos de Deusto 54/ 2016: 40. 
trarse a continuación en la relación entre ésta y la Unión Europea y comparar los estudios de una y otra (III). El centro del trabajo está en torno a la UE como un actor global (IV), en que después de algunas puntualizaciones conceptuales y teóricas, se expone con bastante detalle la Estrategia Global para la Política Exterior y de Seguridad de la Unión Europea de 2016. A continuación se añaden unas breves consideraciones específicas acerca de la Unión Europea como un actor diplomático (V) y, dado que la gobernanza global implica interactuación y cooperación entre una pluralidad de actores públicos y privados, se considera el tema de las percepciones entre ellos (VI). En la conclusión (VII) se resumen algunos puntos de referencia significativos.

\section{Dos procesos del surgimiento de la gobernanza global}

Dos grandes procesos de finales del siglo XX y comienzos del XXI han condicionado e influido el surgimiento del horizonte y la estrategia de la gobernanza global: los poderes emergentes y la globalización.

\section{Los poderes emergentes}

Un poder emergente se define inicialmente como «un actor que está desarrollando capacidades globales y actúa globalmente en la mayoría o todas las dimensiones del poder ${ }^{2}$, de las que se identifican siete: la geografía, la población, los recursos, la economía, la militar, la diplomacia y la identidad. China, India, Brasil y Rusia son los principales nuevos jugadores de esa liga. «El mundo está deviniendo crecientemente multipolar, pero asimétricamente» ${ }^{3}$, con Estados Unidos como el único superpoder de momento. La multipolaridad asimétrica en un espacio limitado y con interconexiones entre los polos apunta recientemente a cierta multilateralidad, también asimétrica, y ésta a un horizonte y posible estrategia de gobernanza global $^{4}$.

2 Thomas Renard, «A Multipolar World in the Making», en The European Union and Emerging Powers in the $21^{\text {st }}$ Century. How Europe Can Shape a New Global Order, ed. por Thomas Renard y Sven Biscop (Farnham: Ashgate, 2012), 56.

3 Renard, «A Multipolar...», 56.

${ }^{4}$ Marc Saxer, «Multilateralism in Crisis? Global Governance in the Twenty-First Century», en The European Union and Emerging Powers in the $21^{\text {st }}$ Century. How Europe Can Shape a New Global Order, ed. por Thomas Renard y Sven Biscop (Farnham: Ashgate, 2012), 61 . 
Europa es la más firme proponente del multilateralismo, teniendo un interés vital en una efectiva gobernanza global, a la que valiosamente puede contribuir con su experiencia supranacional, siempre que reconozca las nuevas realidades y abandone algunos de sus viejos intereses y reflejos. «Europa podría o bien co-determinar las futuras reglas de la gobernanza global o bien encarar un declinar renqueante, marginalización gradual y en el peor caso incluso una irrelevancia total» ${ }^{5}$. Dos cuestiones mayores serán la superación de las crisis de legitimidad interna y de confianza exterior, dos lados de la misma moneda. Se trata de construir una Europa global para la gobernanza global, para lo que Emmanouilidis propone dos prioridades estratégicas: el realce de su rol global y el fortalecimiento de la gobernanza económica europea. El foco en lo nacional y las crecientes desconfianzas mutuas son dos pesados lastres, pero «mirando a las políticas pasadas en respuesta a la globalización, la falta de integración económica y política de la UE aparece incontrovertiblemente como el elefante en la habitación ${ }^{6}$.

Renard y Biscop en las Conclusiones de su libro European Union and Emerging Powers in the 21st Century presentan seis posibles escenarios ${ }^{7}$ : pax americana (EE.UU), pax sínica (China), G 2 (EE.UU.-China), concierto de poderes $($ menor $=5$; mayor $=\mathrm{G} \mathrm{20}$ ), mundo apolar y orden multilateral efectivo, considerando este último el más aceptable y apropiado para el siglo XXI. En él la UE podría ser modelo y líder a lo largo de tres ejes estratégicos: 1) una gran estrategia; 2) un gran acuerdo; y 3) verdaderos partenariados estratégicos.

Las versiones revisadas de las Conferencias Ralph Milliband 20092011, enmarcadas por una Introducción y una Conclusión de Held, acompañado respectivamente por Roger y por Young fueron ordenadas y publicadas en forma de libro en 2013: Global Governance at Risk. En la economía mundial - y no solo en ella - se está dando un cambio (shift) y un choque (shock) con el ascenso de los emergentes (BRICS) ${ }^{8}$, aunque no tan radical y cambiante como con cierta exageración se pensaba hace unos años. Caminamos hacia un «orden mundial emergente describible como una com-

5 Janis A. Emmanouilidis, «Europe's Role in the Twenty-First Century», en The European Union and Emerging Powers in the $21^{\text {st }}$ Century. How Europe Can Shape a New Global Order, ed. por Thomas Renard y Sven Biscop (Farnham: Ashgate, 2012), 83.

6 Andrea Renda, «Globalization, the New Geography of Power and EU Policy Response», en Transworld, Working Paper 10, March 2013, 22.

7 Thomas Renard y Sven Biscop (eds.), The European Union and Emerging Powers in the $21^{\text {st }}$ Century. How Europe Can Shape a New Global Order, (Farnham: Ashgate, 2012), 188-193.

8 Martin Wolf, «The Shift and the Shock: Prospects for the World Economy», en Global Governance at Risk, ed. por David Held y Charles Roger (Cambridge, Polity Press, 2013), 19. 
binación de "incorporación hegemónica" como en el pasado y una nueva "multipolaridad sin multilateralismo"»". Aunque contestados, Occidente y la relación transatlántica (EE.UU.-UE) aún mantienen el liderazgo y la principal responsabilidad en este reto mundial que es la gobernanza. La gobernanza a nivel internacional descansa en cierto grado de cooperación entre Estados más o menos institucionalizada, en el ajuste mutuo de comportamientos conseguido en algún proceso de coordinación de políticas. En un mundo que deviene multipolar y parcialmente multilateral la gobernanza es difícil - ¿es posible? - dada la inercia institucional, favorecida por los dominantes, y la proliferación y fragmentación de regímenes e instituciones.

Para aproximarse a esta gobernanza global se propone una estrategia política que combine una base común (common ground) y una coalición ganadora (winning coalition) de Estados. ${ }^{10}$ La gobernanza global, que implica cierto grado de compromiso y cuyo cumplimiento no puede asegurarse absolutamente, siempre es imperfecta. De aquí la importancia de la construcción de instituciones globales representativas y efectivas, dado que «la capacidad de solución de problemas del existente sistema de instituciones globales, multinacional y transnacional, en muchas áreas no es suficientemente efectiva y responsable para afrontar las crecientes crisis que encaramos» ${ }^{11}$.

\section{La globalización}

En «Globalization and Global Governance» Michael Zürn empieza y acaba reconociendo que los esfuerzos de formulación de una teoría de la interdependencia y de la globalización no han tenido éxito hasta ahora. Distingue entre ambas: la interdependencia se refiere a unidades separadas y es una condición; la globalización apunta a la fusión de unidades y es un proceso. La globalización «describe un proceso en el que el mundo se mueve hacia una sociedad global integrada y disminuye el significado de las fron-

9 Robert H. Wade, «Protecting Power: Western States in Global Organizations», en Global Governance at Risk, ed. por David Held y Charles Roger (Cambridge, Polity Press, 2013), 81 .

10 Benjamin J. Cohen, «The Coming Global Monetary (Dis)Order», en Global Governance at Risk, ed. por David Held y Charles Roger (Cambridge, Polity Press, 2013), 48. David Held y Kevin Young, «From the Financial Crisis to the Crisis of Global Governance», en Global Governance at Risk, ed. por David Held y Charles Roger (Cambridge, Polity Press, 2013), 193.

${ }^{11}$ David Held y Charles Roger (eds), Global Governance at Risk (Cambridge: Polity Press, 2013), 4. 
teras nacionales» ${ }^{12}$. Ese cambio social, desnacionalización societal, puede tener o no desarrollos políticos. Profundiza sobre todo en los efectos más profundos de la globalización - variable independiente - en la gobernanza global - variable dependiente - . La «gobernanza global se refiere a todo el conjunto (entirety) de regulaciones adelantadas para resolver específicos problemas desnacionalizados o proveer bienes comunes transnacionales» ${ }^{13}$. Incluye procesos y estructuras y tiene dos implicaciones importantes: a) es posible la gobernanza más allá del Estado-nación, aunque normalmente falta una autoridad central; y b) se refiere a cierta cualidad de la regulación internacional, presupone algunos intereses comunes y orientaciones más allá del Estado-nación. Todo ello, según Zürn, suscita cuestiones abiertas tales como: supranacionalización, transnacionalización y descentralización; el nuevo rol del Estado y la gobernanza multi-nivel; y problemas estructurales de la gobernanza multi-nivel global respecto a cumplimiento, coordinación, legitimidad, y politización y fragmentación. En la Conclusión se apunta que «la doble demanda de los poderes recientemente emergentes en favor de una participación estatal más igualitaria y de las ONG en favor de una mayor implicación societal en el proceso decisorio internacional pueden ser las fuerzas dirigentes en el desarrollo de la gobernanza global en las próximas décadas» ${ }^{14}$. Está teniendo lugar una transformación de la política mundial, la cual requiere la superación del nacionalismo metodológico.

En Global Governance : Why? What? Whither?, Thomas G. Weiss se pregunta cómo es gobernado el mundo en ausencia de un Gobierno mundial y apunta como respuesta a la gobernanza global, inicialmente definida como «suma de valores, normas, procedimientos e instituciones informales y formales, que ayudan a todos los actores - Estados, organizaciones intergubernamentales (OIGs), sociedad civil, corporaciones transnacionales (CTNs) e individuos - a identificar, comprender y afrontar problemas transfronterizos» ${ }^{15}$. La Unión Europea, indica por otra parte, es un impresionante ejemplo de gobernanza... regional, pues «su relativa cohesión geográfica y cultural limita su aplicabilidad como un modelo global» 16 .

12 Michael Zürn, «Globalization and Global Governance», en Handbook of International Relations, ed. por Walter Carlsnaes, Thomas Risse y Beth A. Simmons (London: Sage, 2013), $402 \mathrm{~b}$.

13 Zürn, Handbook..., 408 a y b.

14 Zürn, Handbook..., 412 b.

15 Thomas G. Weiss, Global Governance: Why? What? Whither? (Cambridge: Polity Press, 2013), 2.

16 Weiss, Global Governance..., 7. 
Tres factores explicarían a juicio del autor la emergencia de la gobernanza global y su arraigo en los ámbitos académicos y políticos en los años 90 del siglo pasado: la interdependencia y los rápidos avances tecnológicos, que hicieron patente que ciertos problemas desafiaban la solución por un único Estado; la expansión numérica y la importancia creciente de los actores no estatales; y una noción de supranacionalidad supuestamente simplista que desconcertaba a muchos analistas. «La gobernanza global representa una casa a medio camino entre la anarquía internacional subyacente al análisis realista y un Estado mundial» ${ }^{17}$. Conviene distinguirla de la «buena gobernanza», la cual incorpora una serie de valores y principios predominantemente occidentales, que la hacen criticable desde diversas sensibilidades. Sí conviene, sin embargo, introducir en ella dos elementos no siempre suficientemente considerados: el poder y la legitimidad, por un lado; y los incentivos, por otro. Pero «la gobernanza global es menos una visión mundial y más una casa a medio camino que proporciona intuiciones adicionales y necesarias cuando intentamos comprender el mundo contemporáneo e identificar nuevas vías de aproximación a las amenazas presentes y futuras» ${ }^{18}$.

Para presentárnosla y acercarnos a ella, Weiss nos proporciona una matriz o retícula con cinco fallas o insuficiencias de la gobernanza global: conocimiento, normas, políticas, instituciones y cumplimiento y seis campos de aplicación o ilustraciones: uso de la fuerza y terrorismo; generaciones de derechos humanos y responsabilidad de proteger; desarrollo humano y cambio climático.

Concluye explicándonos que «la elección de proceder falla a falla fue diseñada para subrayar dos realidades específicas. Primera, el itinerario hacia una mejor gobernanza global consiste en numerosas tareas discretas, pero vinculadas; hemos progresado encarando ciertas fallas en todas las cuestiones, aunque no podemos dormirnos colectivamente en los laureles. Segundo, la especie humana nunca llegará a la solución final de los problemas colectivos del globo» ${ }^{19}$.

Después de haber ido recorriendo de modo muy ilustrativo, sugerente y ponderado los seis campos del uso de la fuerza y del terrorismo, de los derechos humanos y de la responsabilidad de proteger, del desarrollo humano y del cambio climático, a fin de mostrar las fallas e insuficiencias, Weiss se proyecta hacia adelante en el capítulo final: ¿hacia dónde va la gobernanza? Sus respuestas, más bien sugerencias tentativas, son abiertas, flexibles, dinámicas y equilibradas. El mercado no es capaz de asegurar la provisión del bien público definitivo que es la supervivencia con dignidad; por su parte,

17 Weiss, Global Governance..., 25.

18 Weiss, Global Governance..., 41.

19 Weiss, Global Governance..., 170. 
el Estado y las organizaciones intergubernamentales se muestran impotentes ante graves problemas actuales transfronterizos; además, es evidente la falta de una autoridad global central y no parece que vaya a haberla por bastante tiempo. Ante esto, ¿gobernanza global sin Gobierno global para afrontar los problemas globales de la humanidad? Como ha venido repitiendo el autor, la gobernanza global es una casa a medio camino, un puente entre lo viejo y lo aún no nacido: «el núcleo de mi argumento es que numerosas fallas actuales deberían y podrían ser mejor afrontadas con una variedad de modos ... al mismo tiempo estos importantes pasos deberían darse sin abandonar una visión de un futuro mejor ... nuestra tarea inmediata es fusionar idealismo y realismo ... buscar un futuro más ético sin tener en cuenta el poder y los intereses es estúpido, pero el poder y los intereses son ciegos sin un fundamento ético» ${ }^{20}$.

\section{La Unión Europea en la gobernanza global}

En la «Introducción» a The European Union and Global Governance Mario Telo fundamenta la influencia de aquélla sobre ésta en sus logros desde hace tiempo como un modelo de cooperación regional, un actor múltiple y un tipo de poder incipiente y sin precedentes. Hay que tomar en cuenta dos clases de variables: externas e internas, a la hora de valorar su impacto.

Respecto a la dimensión y variables externas «la cuestión más apremiante que ha surgido desde el final de los años 1990 es la medida en que tales modelos de cooperación regional europea ofrecen formas innovadores de actoridad (actorness) y relevancia en un mundo cambiante económica, medioambiental y políticamente ${ }^{21}$, la cual se divide en tres subcuestiones: a) respecto a la primera: ¿un modelo relevante para la gobernanza regional en otros sitios?, la respuesta es muy positiva, pues la UE es un actor relevante para consolidar un tipo multinivel de gobernanza multilateral y puede ser una referencia mundial; b) en relación a la segunda: ¿políticas relevantes de la UE para las organizaciones internacionales y mecanismos de gobernanza global? ¿para combinar el multirregionalismo con un proceso de reforma de las instituciones globales de gobernanza? la respuesta es más ambigua, dados los muy relevantes roles de la UE por un lado, pero cargados de sorprendentes paradojas por otro; y en cuanto a la tercera: ¿es el poder emergente de la UE pertinente para futuras evoluciones del sistema interes-

20 Weiss, Global Governance..., 185.

${ }^{21}$ Mario Telo (ed.), The European Union and Global Governance (London: Routledge, 2009), 15. 
tatal? se deja abierta la contestación al considerar los grandes retos políticos internacionales que surgen dentro de un sistema mundial asimétrico y heterogéneo, que desafían a la gobernanza global.

Se pregunta entonces el autor bajo qué condiciones la UE podría pasar de ser un actor prepolítico y formado a retazos de la gobernanza multinivel global a tener un rol de coliderazgo dentro de un sistema multilateral regional y global, capaz de enmarcar el desorden mundial de la post-guerra fría. No está claro todavía y dependerá de la disposición de los Estados a cooperar y también de la credibilidad, estrategia y capacidades de la UE, pero «solamente un escenario de real progreso en la gobernanza global multilateral y multinivel es compatible con la emergencia de la UE como un líder regional y global o más exactamente como un co-líder» ${ }^{22}$.

La red multilateral post-hegemónica está en un punto crucial: declinar debido al deterioro de su legitimidad y eficiencia o superar la mera forma de gobernanza global para hacerse un principio dirigente de un nuevo orden mundial. Se necesita un nuevo multilateralismo, escenario virtuoso que incluiría a ambos gobernanza y gobierno de los asuntos mundiales y debe ser fomentado mediante una profundización de las actuales experiencias de «reciprocidad difusa», «permitiendo así una incrementada confianza mutua entre Estados miembros» ${ }^{23}$. A la UE le queda mucho por hacer en esta dirección.

Por lo que se refiere a la dimensión y variables internas se señalan tres factores principales: 1) la consolidación de los logros pasados de la cooperación multilateral interna de la UE; 2) la reevaluación de los acuerdos positivos del proceso de ampliación; y 3) la evolución de las capacidades internas de la UE: recursos materiales, base legal, gobernanza práctica.

Wunderlich y Bailey (2011) en la «Introducción» a The European Union and Global Governance. A Handbook exploran la noción de gobernanza global. Es una respuesta a los procesos de globalización más allá del modelo estato-céntrico, la cual incluye a otros actores del escenario global, abarca un amplio espectro de cuestiones y prescinde de una autoridad soberana omniabarcante. Se distingue por cinco características: un sistema multinivel de múltiples estratos (global, internacional, regional, transnacional, nacional y subestatal); un sistema internacional más cooperativo; múltiples actores; complejidad estructural; e integración del nivel y las instituciones regionales en el marco de la gobernanza global. Lee Miles, siguiendo a Baylis y Smith entiende la gobernanza global como «el sistema evolutivo de coordinación política formal e informal, a través de múltiples

22 Telo, The European Union..., 25.

23 Telo, The European Union..., 29. 
niveles desde el local al global - entre autoridades públicas (Estados y organizaciones intergubernamentales) y agencias privadas (ONGs y actores corporativos), que busca manejar problemas colectivos mediante la elaboración e implementación de normas, reglas, programas y políticas globales o transnacionales ${ }^{24}$. La gobernanza global trata de afrontar los retos de la interdependencia global.

Warleigh-Lack ha examinado la relación de los estudios de la integración regional europea con los de la gobernanza global. Es necesario que los primeros se abran a ésta y aprendan de ella, si no quieren hacerse obsoletos en un mundo al que la globalización ha cambiado y donde está generando una 'politeia global'. Los estudios de la integración europea se deben abrir y comprometer activamente con la gobernanza global para «comprender la Unión más holísticamente, i. e. como una entidad 'co-constituida por ambas sus acciones y reacciones en el medio global' (Manners, 2003, 77)» 25 .

Además, los estudios de la integración europea, innovadores en no pocos aspectos de las actuales relaciones internacionales, también puede enseñar no poco a la gobernanza global. En esta dirección Wunderlich destaca dos fortalezas particulares de los estudios de integración europea: primera, la gobernanza multinivel y las conceptualizaciones en red; y segunda, la actoridad (actorness) más allá del Estado. Hay que tener en cuenta también que la experiencia de la gobernanza multinivel de la Unión Europea, con sus logros y fracasos en el siglo XXI, puede ayudar a reflexionar y tal vez prevenir algunas de las dificultades de la futura gobernanza global, especialmente en situaciones de crisis. «Para resumir, las aproximaciones de la gobernanza multinivel ofrecen un punto de vista analítico y una estrategia no solo para el sistema de elaboración de políticas de la UE, sino también para el complejo contemporáneo de la gobernanza global» ${ }^{26}$.

Las teorías de la integración europea y las de la gobernanza global tienen elementos similares en parte y también notables diferencias, que Miles ha analizado sutílmente. Entre los puntos de partida comunes indica: 1) el manejo de los retos de la interdependencia, aunque lo hagan en un nivel regional las primeras y en uno global las otras; 2) un rol para el Estado-nación reactualizado y transformado; 3) la difuminación de las competencias con

${ }^{24}$ Lee Miles, «When a Fusing Europe and a Globalizing World Meet», en The European Union and Global Governance. A Handbook, ed. por Jens-Uwe Wunderlich y David J. Bailey (London: Routledge, 2011), 20.

25 Alex Warleigh-Lack, «Obsolete if Obstinate? Transforming European Union Studies in the Transnational Era», en The European Union and Global Governance. A Handbook, ed. por Jens-Uwe Wunderlich y David J. Bailey (London: Routledge, 2011), 17.

26 Jens-Uwe Wunderlich, «European Integration, Global Governance and International Relations», en The European Union and Global Governance. A Handbook, ed. por Jens-Uwe Wunderlich y David J. Bailey (London: Routledge, 2011), 51. 
cambios de ubicación y de poder; y 4) la tendencia a la privatización. En cuanto a las diferencias señala las espaciales y las temporales. Respecto al espacio Miles apunta a la integración europea como un proceso gobernado por valores y normas y por reglas dictadas por las elites políticas tradicionales mientras la gobernanza global apunta al espacio virtual y a una nueva sociedad civil global más flexible e informal. En relación con el tiempo la integración europea enfatiza la continuidad y el progreso gradual de un proceso evolutivo a largo plazo en tanto las aproximaciones de la gobernanza global son más sensibles a procesos decisorios flexibles y responsivos que operan e influyen a corto plazo fuera del control de las elites políticas tradicionales.

\section{La UE un actor global}

\section{Presencia $y$ «actoridad» (actorness)}

En 1999 Bretherton y Vogler publicaron The European Union as a Global Actor, cuya segunda edición (2006) seguía manteniendo el foco en la capacidad del actor, afrontando además su naturaleza y la medida en que la UE se había hecho un actor en la política global.

El cap. I trataba de conceptualizar los actores y la «actoridad» o capacidad del actor (actorness): «implica una entidad, que exhibe un grado de autonomía de su entorno externo y también de sus constituyentes internos, y que es capaz de volición y propósito» ${ }^{27}$. Los autores se aproximan a la UE como «un actor en construcción», considerando una serie compleja de procesos interactivos basados en las nociones de oportunidad (contexto estructural), presencia (poder estructural de la UE) y capacidad (contexto interno de la acción externa de la UE), que se coordinan de diversos modos para configurar actividades externas de la UE. Esos tres elementos o factores: oportunidad, presencia y capacidad constituyen la «actoridad».

El cap. 2 se ocupa de la construcción de la identidad colectiva de la Unión y de sus roles asociados, que influyen sobre las prácticas hacia terceros. La identidad se logra en el curso de la interacción social y está constituida por comprensiones compartidas, presentando la de la UE dos caras: inclusiva y exclusiva. Hay numerosas áreas de identidad híbrida asociada con tensiones e inconsistencias sin que haya, sin embargo, necesariamente una contradicción fundamental.

27 Charlotte Bretherton y John Vogler, The European Union as a Global Actor (London: Routledge, $20062^{\text {nd }}$. ed.), 16. 
Los capítulos 3 al 8 exploran la «actoridad» global de la Unión en diferentes áreas de políticas, lo que Barbé califica como «una especie de actorness a varias velocidades» ${ }^{28}$.

Bretherton y Vogler concluyen su obra calificando a la Unión Europea como «un sistema político en construcción» en una cambiante situación internacional.

Otros autores siguieron acercándose en el primer decenio del siglo XXI al estudio de la capacidad de actor global de la UE desde diversas perspectivas: mayor integración económica y política de ésta, inter-regionalismo internacional, la Unión como imperio diferente, ejemplo o modelo de poder, superpoder ilustrado, etc...

Con fuerte acento globalizante escribe en 2010 Howorth el artículo «The EU as a Global Actor: Grand Strategy for a Global Grand Bargain?», en el que propone que la UE alcance su grandeza (EU achieving greatness), en el sentido de «influencia e impacto en un mundo crecientemente turbulento y en rápido cambio... forjando acuerdos multilaterales que puedan conducir la historia hacia vías cooperativas y consensuales» ${ }^{29}$, lo que Hutchings bautizó como un «gran acuerdo global» («global grand bargain»). La Unión Europea no puede considerarse un poder mundial en sentido convencional, pero existen poderosos factores que avalan su potencial de influir en una dirección positiva: «lo que la UE debería buscar como un objetivo estratégico básico es un mundo de diversidad cultural y política en el que, sin embargo, la estabilidad, la seguridad, la prosperidad, el desarrollo, la sostenibilidad medioambiental, la solidaridad y la autodeterminación fueran considerados en términos holísticos como elementos claves de la interdependencia global - de la inter-polaridad» ${ }^{30}$.

En tiempos y espacios de globalización se ha ido elaborando el horizonte y estrategia de la gobernanza global, en la que la Unión Europea quiere ser una activa configuradora y aportar además su experiencia, en una palabra llegar a ser un actor global. En esa dirección Hettne distingue entre los conceptos de actorship, más estructural, y de actorness, más cercano al de agencia, que aquí nos permitimos traducir respectivamente como «actoría» $\mathrm{y}$ «actoridad». El autor elabora después un poco más este último concepto: «La actoridad (actorness) - refiriéndose generalmente al comportamiento externo - implica un blanco de la acción y un espacio de maniobra,

${ }^{28}$ Esther Barbé (dir.), La Unión Europea en las Relaciones Internacionales (Madrid: Tecnos, 2014), 24.

29 Jolyon Howorth, «The EU as a Global Actor: Grand Strategy for a Global Grand Bargain», Journal of Common Market Studies 48:3 (2010), 457.

${ }^{30}$ Howorth, «The EU as a Global ...», JCMS 48:3 (2010),469. 
en algunos casos incluso una personalidad legal» ${ }^{31} \mathrm{e}$ indica que la gran cuestión es la transformación real de la fuerte presencia internacional de Europa en la capacidad intencional de configurar el entorno exterior influyendo en otros actores y en última instancia en el orden mundial, es decir, el paso de la actoría (actorship) a la actoridad (actorness).

\section{Estrategias europeas globales}

Desde hace varias décadas y en distintos ámbitos existe una tradición de estudios sobre el probable contexto mundial y posible acción futura de las CCEE/UE: informes, proyectos, perspectivas, grupos de reflexión, escenarios, estrategias. Esa línea ha continuado en los cambiantes tiempos y espacios de comienzos del siglo XXI. El estudio de la Unión Europea como un actor global ha sido controvertido. Schunz ${ }^{32}$ recuerda su evolución desde los debates iniciales a comienzos del siglo XXI en torno a la «presencia» $\mathrm{y}$ «actoridad» («actorness») de la UE en los asuntos mundiales a la posterior investigación sobre su capacidad y el tipo de actor global: «poder civil», «poder normativo», «modelo», «líder», «imperio». Otra línea ha primado el intento de una mayor comprensión del contexto exterior en el que opera la Unión Europea y las correspondientes adecuaciones, destacando la necesidad de una acción más estratégica ${ }^{33}$. A continuación se expone resumidamente Shared Vision, Common Action: A Stronger Europe. A Global Strategy for the European Union's Foreign and Security Policy ${ }^{34}$. En el Consejo Europeo de 25 y 26 de junio de 2015 se había acordado que la Unión preparara una nueva estrategia que la alta representante Federica

31 Björn Hettne, «The European Union as an Emerging Global Actor», en The European Union and Global Governance. A Handbook, ed. por Jens-Uwe Wunderlich y David J. Bailey (London: Routledge, 2011), 31.

32 European Commission, A Global Actor in Search of a Strategy. European Union Foreign Policy between Multilateralism and Bilateralism (EUR 26572 EN) (autor: Simon Schunz) (Bruxelles: European Union, 2014), 10-11.

${ }^{33}$ En mi artículo «Globalización-Unión Europea-Globalización», Cuadernos Europeos de Deusto 54/2016, 52-58 pueden leerse exposiciones resumidas de Una Europa Segura en un Mundo Mejor. Estrategia Europea de Seguridad (Consilium 2003), Proyecto Europa 2030. Retos y Oportunidades. Informe al Consejo Europeo (Grupo de Reflexión sobre el Futuro de la UE en 2030, 2010), Towards a European Global Strategy. Securing European Influence in a Changing World (Istituto Affari Internationali et al., 2013) y A Global Actor in Search of a Strategy. European Union Foreign Policy between Multilateralism and Bilateralism (European Union 2014).

${ }^{34}$ European Union, Shared Vision, Common Action: A Strong Europe. A Global Strategy for the European Union's Foreign and Security Policy, (June 2016), <http://europa.eu/ globalstrategy/en>. 
Mogherini habría de presentar a más tardar en la cumbre de 2016, como así fue.

En contraste con la percepción optimista de la Unión Europea y de su posición en el mundo de la Estrategia Europea de Seguridad de $2003^{35}$, la Estrategia Global de 2016 parte de una valoración crítica e incluso dramática: «vivimos en tiempos de crisis existencial, dentro y más allá de la Unión Europea. Nuestra Unión está amenazada» ${ }^{36}$. También son tiempos de extraordinarias oportunidades. Entre esas amenazas y oportunidades tendrá que navegar una Europa más fuerte, que piense estratégicamente, comparta una visión y actúe junta, guiada por sus intereses, principios y prioridades.

La globalidad de la Estrategia, como explica en el prefacio la alta representante Federica Mogherini, no tiene precisamente sentido geográfico, sino que se refiere más bien a la amplia gama de políticas e instrumentos que promueve la Estrategia: poder blando y duro, autonomía estratégica y sistema internacional basado en reglas y en el multilateralismo, Europa unida y comprometida con la diversidad.

La primera parte está dedicada a la promoción de los intereses de nuestros ciudadanos, indicando que van mano a mano con nuestros valores y destacando los vitales, sustentadores de nuestra acción exterior, y sus medios: la paz y seguridad requieren las capacidades para defendernos y cumplir nuestros compromisos de asistencia mutua y solidaridad; la prosperidad de la Unión es base de una Europa más fuerte y también contribución a las metas del desarrollo sostenible en el nivel mundial; la democracia resiliente: «viviendo consistentemente según nuestros valores internamente determinará nuestra credibilidad e influencia externas» ${ }^{37}$; y un orden global basado en reglas, cuyo principio clave es el multilateralismo y su núcleo las Naciones Unidas.

La segunda parte se ocupa de los principios que guían nuestra acción exterior. Deben de ser claros, combinación de evaluación realista e inspiración idealista, responsables hacia los otros y sensibles a las contingencias, en resumen un «pragmatismo con principios» (principled pragmatism). Se desatacan cuatro: la unidad, que nunca ha sido tan vital y urgente, pues solo juntos tendremos el suficiente potencial; el comprometimiento a participar plenamente en el mercado global y co-configurar sus reglas; la responsa-

35 Consilium, Una Europa Segura en un Mundo Mejor. Estrategia Europea de Seguridad (Bruselas, 2003). <http:www.consilium.europa.eu/uedocs/cmsUpload/031208ESSIIES.pdf>. La Estrategia Europea de Seguridad de 2003 comenzaba con las memorables frases «Europa no ha sido nunca tan próspera, tan segura, ni tan libre», «La creación de la Unión Europea ha sido un factor esencial de esta evolución».

${ }^{36}$ European Union, Shared..., 13.

37 European Union, Shared..., 15. 
bilidad especialmente en Europa y sus regiones circundantes, con comprometimientos por objetivos en espacios más alejados; y el partenariado, de modo que la corresponsabilidad será nuestro principio guía.

La parte tercera de la Estrategia Global presenta cinco grandes prioridades de la acción exterior de la Unión Europea:

\section{La seguridad de nuestra Unión}

Hoy la UE afronta grandes amenazas desde el cambio climático al terrorismo. Es esencial cambiar el paso. «Debemos trasladar nuestros comprometimientos a la asistencia mutua y solidaridad en la acción y contribuir más a la seguridad colectiva de Europa ${ }^{38}$. Para ello se proponen cinco líneas de acción: a) seguridad y defensa. «Debemos estar preparados y ser capaces de disuadir, responder y protegernos» contra las amenazas externas e internas para lo que se necesitan medios tecnológicos e industriales, esfuerzo concertado y operativo, investigación e industria de defensa; b) contraterrorismo. Incremento de la inversión, solidaridad, participación de la información y cooperación en inteligencia. «Lo más crucial de todo, la UE vivirá de acuerdo a sus valores interna y externamente: éste es el antídoto más fuerte que tenemos contra el extremismo» ${ }^{39}$; c) ciberseguridad; d) seguridad energética interna y externa; y e) comunicaciones estratégicas.

\section{Resiliencia estatal y societal a nuestro Este y Sur}

La fragilidad cerca de nuestras fronteras amenaza nuestros intereses vitales. «En contraste, la resiliencia - la capacidad de los Estados y sociedades para reformar, resistiendo y recuperándose así de las crisis internas y externas - nos beneficia a nosotros y a los países en nuestras regiones circundantes... Por tanto la UE, junto con sus compañeros promoverá la resiliencia en sus regiones circundantes $»^{40}$. «Una sociedad resiliente caracterizada por la democracia, la confianza en las instituciones y el desarrollo sostenible subyace en el corazón de un Estado resiliente» ${ }^{41}$. Política de ampliación. Nuestros vecinos. Resiliencia en nuestras regiones circundantes. Una política de migración efectiva.

38 European Union, Shared..., 19.

39 European Union, Shared..., 21.

40 European Union, Shared..., 23.

41 European Union, Shared..., 24. 


\section{Una aproximación integrada a conflictos y crisis}

Los conflictos y crisis amenazan nuestros intereses vitales compartidos. La Unión Europea se concentrará en sus regiones circundantes al Este y al Sur, considerando caso por caso su implicación más lejos. Puesto que todos esos conflictos se caracterizan por sus múltiples dimensiones, se hace necesaria una «aproximación comprehensiva» de múltiples fases, múltiples niveles y multilateral. «La UE fomentará la seguridad humana mediante una aproximación integrada» ${ }^{42}$ : a) paz preventiva: alerta y acción tempranas; b) seguridad y estabilización: mejor equipamiento para construir la paz, garantizar la seguridad, proteger las vidas humanas, ayudar a combatir el terrorismo y apoyar a las legítimas instituciones a prestar servicios básicos y seguridad a las poblaciones locales en casos de estabilización; c) solución de conflictos: «cada país con conflicto necesitará reconstruir su propio contrato social entre el Estado y sus ciudadanos. La Unión apoyará tales esfuerzos» ${ }^{43}$; y d) economía política de la paz: mayores sinergias entre la asistencia humanitaria y al desarrollo. Medidas restrictivas y diplomacia para el cambio pacífico.

\section{4. Órdenes regionales cooperativos}

La Estrategia Global formula una firme y elocuente fundamentación de una gobernanza global de base regional en la que la Unión Europea juega y debe seguir jugando una baza clave: «En un mundo cogido entre presiones globales y retrocesos locales, la dinámica regional viene al primer plano... Las regiones representan espacios críticos de gobernanza en un mundo descentrado... Ésta es una razón fundamental para la paz y el desarrollo de la propia UE en el siglo XXI... Los órdenes regionales no toman una única forma... En diferentes regiones del mundo, la UE será guiada por objetivos específicos» ${ }^{44}$. A continuación se hace un breve recorrido específico por las grandes regiones del mundo y las diversas políticas de la UE respecto a ellas: Rusia; Mediterráneo, Oriente Próximo y África; Atlántico Norte y Sur; Asia; y finalmente el Ártico.

\section{Gobernanza global para el siglo XXI}

Sin normas globales y los medios para imponerlas, la paz, la seguridad, la prosperidad y la democracia, intereses vitales de la Unión Europea, co-

42 European Union, Shared..., 28.

43 European Union, Shared..., 30-31.

${ }^{44}$ European Union, Shared..., 32. 
rren peligro. «La UE está comprometida con un orden global basado en el derecho internacional, incluyendo los principios de la Carta de las NN.UU... Este comprometimiento traslada a una aspiración la transformación del sistema existente más que la simple preservación» ${ }^{45}$. A continuación la Estrategia Global se refiere con algún detalle a la reforma, inversión, implementación, profundización, ensanchamiento, desarrollo y partenariado para la gobernanza global en el siglo XXI.

La parte cuarta de la Estrategia Global para la Política Exterior y de Seguridad de la Unión Europea trata del paso de esta visión que ha expuesto a la acción: «perseguiremos nuestras prioridades movilizando nuestras redes sin igual, nuestro peso económico y todos los instrumentos a nuestra disposición de un modo coherente y coordinado. Para cumplir nuestras metas, sin embargo, debemos invertir colectivamente en una Unión creíble, responsiva y conjuntada» ${ }^{46}$ : a) una Unión creíble. La credibilidad es esencial y el poder blando no es suficiente, sino que hay que fortalecerla en seguridad y defensa: valoración mejor y compartida de las amenazas y retos internos y externos; inversión en capacidades digitales; capacidades militares; industria de defensa. «Los Estados miembros necesitarán moverse hacia la cooperación en defensa como norma» ${ }^{47}$; b) una Unión responsiva. Lo único predecible es la impredecibilidad, lo desconocido está adelante. Una Unión más responsiva requiere cambio: acción diplomática completamente fundada en el Tratado de Lisboa; más rápida y efectiva política común de seguridad y defensa; política de desarrollo más flexible y alineada con nuestras prioridades estratégicas; c) una Unión conjuntada, a través de nuestras políticas internas y externas.

Una política hacia adelante. «Esta Estrategia se sustenta en la visión y la ambición de una Unión más fuerte... Ahora debemos trasladarla rápidamente en acción» ${ }^{48}$ con las pertinentes revisiones e innovaciones futuras.

En el Consejo Europeo de junio de 2016 se presentó esta Estrategia Global (Consejo Europeo, 28 de junio de 2016, III, 20), por la que éste se congratuló e invitó a seguir la labor. Fue, sin embargo, recibida con frialdad y no poco escepticismo en un momento particularmente problemático de la Unión Europea y de su entorno inmediato: Brexit, crisis de los refugiados, Ucrania, Siria, etc. Después de analizar su texto y contexto, Arteaga ${ }^{49}$ concluye matizadamente acerca de su desarrollo y potencialidades: «Aun-

45 European Union, Shared..., 39.

46 European Union, Shared..., 44.

${ }^{47}$ European Union, Shared..., 45.

48 European Union, Shared..., 51.

49 Félix Arteaga, «La defensa europea entre la Estrategia Global y su implementación». Documento de trabajo 16/2016, (Madrid: Real Instituto Elcano, 2016), 17. 
que no se ha elaborado ninguna estrategia sectorial, el desarrollo de la Estrategia está conciliando sin grandes dificultades por el momento las distintas iniciativas de implementación... Si todo va como se espera, y el proceso de desarrollo no se ve afectado por las graves circunstancias que afectan al proceso europeo de integración, la implementación de la Estrategia puede potenciar, aunque a medio plazo, una mayor autonomía estratégica de la UE. De este desarrollo se beneficiará tanto el vínculo trasatlántico (equilibrando cargas y responsabilidades entre las dos orillas del Atlántico) como el nexo exterior-interior (integrando los procesos de internacionalización e internalización de ambas esferas de seguridad)».

\section{La Unión Europea como un actor diplomático}

Desde el Tratado de Maastricht (1992), que instituyó la Unión Europea y la abrió a la Política Exterior y de Seguridad Común (PESC), el papel de ésta como actor internacional se ha venido incrementando en el mercado, en la seguridad, como actor político y actor normativo, lo que conllevó una creciente necesidad y demanda de llegar a ser también un actor diplomático. El Tratado de Lisboa (2007), después de sus grandes dificultades de elaboración y aprobación, así como de muy significativos recortes, fue otro hito clave: «El texto jurídico sobre la acción exterior de la Unión del Tratado de Lisboa varía poco respecto al innovador del TC [Tratado Constitucional], pero el contexto político es otro, crecientemente intergubernamental. La matización de la forma jurídica tiene causas políticas de fondo y una y otro probablemente tendrán también efectos políticos en los Estados miembros y en los ciudadanos. Es el círculo poco virtuoso del déficit de europeísmo ${ }^{50}$. De aquí que los primeros años posteriores estuvieran marcados por los arreglos domésticos, pero el mundo seguía adelante y planteaba enormes desafíos, que hacían más y más necesaria la diplomacia de la Unión Europea.

Joachim A. Koops y Gjovalin Macaj en su «Introducción: la Unión Europea como un actor diplomático» adoptan una perspectiva multi-nivel y multi-actor: actores claves en los niveles individual, de las organizaciones, de los Estados miembros e internacional; se incluye también el juego entre las propias instituciones de la UE, así como entre éstas y las políticas de algunos Estados miembros. «La perspectiva de "la UE como un actor diplomático" se centra en la capacidad de la UE para comprometerse autoritati-

\footnotetext{
${ }^{50}$ Nicolás Mariscal, Más allá de Lisboa: Horizontes Europeos (Madrid, Tecnos, 2010), 180 .
} 
vamente en el proceso de negociación, representación y comunicación a fin de influir en terceras partes» ${ }^{51}$.

Desde 1989, con el final de la Guerra Fría y según algunos también del siglo XX, se abre un tiempo nuevo con profundos cambios, que van a exigir también los de la diplomacia. Smith señala tres: 1) la estructura del sistema: de la bipolaridad a la unipolaridad y finalmente a la multipolaridad actual; 2) la estructura de oportunidad global con el fenómeno de la hibridación, «por el que los espacios diplomáticos se han abierto y han sido 'invadidos' por diferentes actores e influencias y ha habido una unión de diversas series de reglas y prácticas», ${ }^{52}$ y 3 ) la función de «gestión» («management») que se ha hecho más difícil por la dimensión estratégica de la interacción, la diplomacia estructural y la diplomacia transformadora.

En este contexto más amplio en el espacio y en el tiempo hay que situar también la diplomacia de la UE, que continúa planteando cuestiones de legitimidad interna y externa.

A la hora de evaluar el impacto de la diplomacia de la Unión Europea, Kleistra y Van Willigen ${ }^{53}$ indican una serie de riesgos y los correspondientes desafíos para superarlos: ante la falta de clara distinción al medir la efectividad, la actuación y el impacto, abogan por una más específica y precisa definición de este último; ante la estrechez instrumental, por una percepción de la diplomacia UE como un proceso multinivel; ante la homogeneidad después de Lisboa, por la consideración de la heterogeneidad de actores y preferencias; frente a una aproximación líneal, por un proceso diplomático caracterizado por la causalidad compleja y la volatilidad; y ante la no distinción entre diferentes marcos diplomáticos, actividades diplomáticas y áreas políticas, abogan por distinguirlos y diferenciarlos.

Finalmente, no es posible cerrar el tema sobre la diplomacia de la Unión Europea en la gobernanza global sin mencionar siquiera el rol del Servicio Europeo de Acción Exterior, sobre el que existe ya una amplia bibliografía especializada ${ }^{54}$.

51 Joachim A. Koops y Gjovalin Macaj, «Introduction: The European Union as a Diplomatic Actor», en The European Union as a Diplomatic Actor, ed. por Joachim A. Koops y Gjovelin Macaj (Basingstoke: Palgrave Macmillan, 2015), 2.

52 Michael H. Smith, «The EU as a Diplomatic Actor in the Post-Lisbon Era: Robust or Rootless Hybrid», en The European Union as a Diplomatic Actor, ed. por Joachim A. Koops y Gjovelin Macaj (Basingstoke: Palgrave Macmillan, 2015), 16.

53 Yvonne Kleistra y Niels Van Willigen, «Evaluating the Impact of EU Diplomacy: Pitfalls and Challenges», en The European Union as a Diplomatic Actor, ed. por Joachim A. Koops y Gjovelin Macaj (Basingstoke: Palgrave Macmillan, 2015).

54 Knud Erik Jorgensen, «EU Diplomacy in Global Governance: The Role of the European External Action Service», en The European Union as a Diplomatic Actor, ed. por Joachim A. Koops y Gjovelin Macaj (Basingstoke: Palgrave Macmillan, 2015), 31-51. 


\section{La percepción de la UE por otros actores de la gobernanza global}

La gobernanza global, según la hemos conceptualizado, es un proceso y una estrategia, en los que una pluralidad de actores públicos y privados de diversos niveles interactúan y cooperan, sin un Gobierno común, para manejar problemas específicos desnacionalizados o proveer bienes públicos transnacionales. En esa interactuación y cooperación entre los actores cuentan las percepciones entre ellos. En nuestro caso específico las que la UE tenga de los otros y las que éstos tengan de ella. Estas segundas percepciones son las que aquí nos interesan sobre todo, porque influirán en su actuación respecto a la Unión Europea; además esa imagen externa de la UE contribuirá a configurar en parte la identidad y auto-imagen que ésta se haga de sí misma; y también en parte su comportamiento como actor regional y global.

Desde los comienzos del siglo XXI se han multiplicado las investigaciones acerca del impacto de la imagen externa de la Unión Europea sobre su política exterior, pero se han llevado a cabo desde múltiples aproximaciones disciplinarias que raramente dialogan y se cruzan, de modo que «todavía tiene que emerger un modelo teórico abarcante unificador» ${ }^{55}$. Chaban y Holland van a tratar de contribuir a esa tarea.

Distinguiéndolo de las imágenes, categorías y estereotipos, van a seguir un concepto de percepción como «el resultado de la cognición subjetiva o psicológica del observador, más que el reflejo objetivo del objeto observado (Shiming, 2010, p. 269)» ${ }^{56}$. Las percepciones son específicas para diversas cuestiones, localizaciones, tiempos y cohortes. Además las percepciones de los actores internacionales constituyen complejas constelaciones de significados configuradas por numerosos factores interactuantes.

Buscando un modelo unificador de las percepciones externas sobre la UE van a seguir la teoría de la otredad (theory of Othering) de Pickering en 2001. «En el núcleo de este modelo está el concepto del Otro, que considera "ambos, aquéllos implicados en el proceso de otredad, así como también el objeto de este proceso" (Pickering 2001, 69)» ${ }^{57}$. La UE es vista como el Otro del mundo externo, es hecha el Otro de los otros, otredad de la UE para diversos actores externos.

Señalan a continuación cuatro posibles escenarios de la intersubjetividad en el diálogo de la Unión Europea y el mundo: 1) aprecio y respeto de

55 Natalia Chaban y Martin Holland, «Introduction: The Evolution of EU Perceptions: From Single Studies to Systemic Research», en Communicating Europe in Times of Crisis. External Perceptions of the European Union, ed. por Natalia Chaban y Martin Holland (Basingstoke: Palgrave Macmillan, 2014), 6.

56 Chaban y Holland, Communicating..., 8.

57 Chaban y Holland, Communicating..., 11. 
la UE como un actor global mayor; 2) cambios de identidad entre los receptores internacionales de los mensajes de la UE; 3) negación de la UE y de sus acciones internacionales; y 4) rechazo activo y visiones anti-UE. Se apunta un potencial quinto escenario de aprendizaje en la interacción SelfOther, que no parece ajustarse rigurosamente a la teoría de la otredad. ¿Qué Otro es la Unión Europea para los otros, para el mundo externo?

Existen distintas aproximaciones y métodos de investigación, señalando Chaban y Holland como los más populares: a) los estudios de países singulares: visiones de la UE desde los Otros estratégicos (China, India, Japón, Rusia, Estados Unidos...); y b) los estudios comparados, que añaden las dificultades de su complejidad y costo. También se llevan a cabo estudios de las percepciones externas sobre la UE a través de diversos tipos de «discursos».

La Unión Europea se ve a sí misma como un actor cualitativamente distinto en la política mundial, mientras ha mostrado poco interés y escucha de lo que el resto del mundo opinaba sobre ella. Fioramonti en «Is the EU a "Better" Global Player?» estudia las percepciones desde los países emergentes. La mayoría de su población conoce poco o nada de la Unión Europea y duda de sus buenas intenciones y efectividad. Las elites políticas, por su parte, aprueban generalmente la aproximación de la UE a la democracia y a la paz, así como el énfasis en el multilateralismo, aunque éste es entendido de otro modo, próximo a la multipolaridad. Puede decirse que hay una visión más positiva de la dimensión política que de la económica y comercial: proteccionismo, doble medida, estrategias amenazantes, imposición de prácticas dudosas, desequilibrio en la distribución del poder internacional. Fioramonti concluye señalando "la significativa "brecha" (significant "gap") entre cómo la UE se percibe a sí misma y cómo es percibida por los países emergentes. Parece que la "singularidad" de la UE como un actor global transido de valores como la solidaridad, sostenibilidad y justicia, que son más bien comunes en la retórica oficial de la UE, es desafiada por la valoración de conjunto hecha por las economías emergentes» ${ }^{58}$.

En «The EU's Leadership in Global Governance: Perceptions from the Others» Lucarelli evalúa «en qué medida la UE es percibida como un líder en la gobernanza global». Señala cuatro requisitos - más importantes en términos subjetivos que objetivos - para ser un líder: 1) recursos de poder (económico, militar, político, «ideacional»); 2) habilidades de negociación; 3) credibilidad; y 4) legitimidad. ¿En qué medida la Unión Europea es per-

${ }^{58}$ Lorenzo Fioramonti, «Is the EU a 'Better' Global Player? An Analysis of Emerging Powers' Perceptions», en The European Union and Emerging Powers in the $21^{\text {st }}$ Century. How Europe Can Shape a New Global Order, ed. por Thomas Renard y Sven Biscop (Farnham: Ashgate, 2012),159. 
cibida teniendo estas cualidades? Apunta previamente Lucarelli a la utilidad de distinguir entre las percepciones respecto a los requisitos de liderazgo y las habilidades reales de liderazgo para hacerlos efectivos y a continuación evalúa las percepciones respecto a los primeros. En cuanto a los recursos de poder es incuestionable el amplio reconocimiento de la UE como un poder económico; también es tenida en cuenta por los Estados y las organizaciones multilaterales en cuestiones de seguridad; se percibe ejemplar la unidad de países que han superado el conflicto violento; también su integración regional, sus valores y la promoción del multilateralismo, aunque en estos últimos casos con matizaciones y críticas. Sus habilidades de negociación son objeto de una percepción ambivalente: positiva, aunque a veces se muestra demasiado débil en el uso del «soft power», y demasiado impositiva e incluso neocolonial otras con condicionamientos a valores eurocéntricos. La credibilidad y la legitimidad de la Unión Europea son afectadas negativamente por sus dobles estándares, inconsistencias y posición subordinada a Estados Unidos. Concluye que hoy no se le reconoce a la Unión Europea un rol de líder en la gobernanza global y se predice aún menor en el futuro ${ }^{59}$ y señala como la más chocante característica de esa incapacidad «la brecha (gap) entre el reconocimiento del gran potencial para el liderazgo (incluyendo una capacidad para una postura innovadora en la política internacional) y el liderazgo real» ${ }^{60}$.

Holland y Chaban en las Conclusiones de su libro se preguntan cómo es vista la UE en los países terceros y en qué medida es influyente para establecer agendas globales y responden apuntando al declinar de las «expectativas y percepciones de la capacidad de la UE para ser un actor multilateral efectivo, uno que es menos capaz y está menos dispuesto a comprometerse globalmente debido a las apremiantes, pero miopes, prioridades locales» ${ }^{61}$. Añaden algunas matizaciones a las evidencias empíricas presentadas en los capítulos precedentes e incluyen algunas sugerencias politológicas y políticas para intentar alterar esas percepciones, concluyendo: «la presencia de la UE no es aún ubicua o ampliamente respetada de necesidad. La indiferencia mezclada con la decepción sigue prevaleciendo a pesar de dos décadas de política exterior y de seguridad común y de la marca "UE". Esta percep-

59 Sonia Lucarelli, «The EU's Leadership in Global Governance: Perceptions from the Others», en Communicating Europe in Times of Crisis. External Perceptions of the European Union, ed. por Natalia Chaban y Martin Holland (Basingstoke: Palgrave Macmillan, 2014), 56.

${ }^{60}$ Lucarelli, Communicating..., 57.

${ }^{61}$ Martin Holland y Natalia Chaban, «Conclusions: Perceptions, Prisms, Prospects», en Communicating Europe in Times of Crisis. External Perceptions of the European Union, ed. por Natalia Chaban y Martin Holland (Basingstoke: Palgrave Macmillan, 2014), 249. 
ción puede cambiar, pero la UE necesita ser reflectiva, centrada y proactiva si desea configurar más efectivamente cómo es percibida. Si fracasa en hacerlo, el propio proyecto de integración puede llegar a estar crecientemente envuelto en la duda, dentro y más allá de las fronteras de la UE. Las percepciones cuentan» ${ }^{62}$.

\section{A modo de conclusión}

De un artículo introductorio y holístico no pueden esperarse conclusiones empíricas, pero sí algunas ideas potencialmente fecundas teórica o aplicadamente. Por tanto aquí, más que conclusiones, se presenta un resumen de puntos de referencia significativos.

1. El mundo se está haciendo multipolar, pero asimétricamente. Esa multipolaridad asimétrica apunta a una multilateralidad también asimétrica y ésta a una posible gobernanza global.

2. La gobernanza global es un horizonte y una estrategia en respuesta a los procesos de globalización.

3. En un mundo que deviene multipolar y parcialmente multilateral, la gobernanza global es difícil, dada la inercia institucional y la proliferación y fragmentación de regímenes e instituciones. La gobernanza global siempre es imperfecta.

4. «La gobernanza global se refiere a todo el conjunto de regulaciones adelantadas para resolver problemas desnacionalizados o proveer bienes comunes transnacionales» (Zürn). Gobierna el mundo en ausencia de un Gobierno mundial. Es «una casa a medio camino» (Weiss) entre la anarquía internacional y un Estado mundial. El itinerario hacia ella consiste en numerosas tareas discretas, pero vinculadas. La especie humana nunca llegará a la solución final de los problemas colectivos del globo.

5. Europa es la más firme proponente del multilateralismo, teniendo un interés vital en una efectiva gobernanza global, pero su insuficiente integración económica y política es su mayor carencia.

6. Algunos autores (Bretherton y Vogler, Hettne) distinguen entre los conceptos de actorship, más estructural, y de actorness, más cercano a la agencia, que nos hemos permitido traducir respectivamente por «actoría» $\mathrm{y}$ «actoridad». La gran cuestión para Europa es el paso de la actoría a la actoridad.

62 Holland y Chaban, Communicating..., 252. 
7. Shared Vision, Common Action: A Stronger Europe. A Global Strategy for the European Union's Foreign and Security Policy de 2016 parte de una valoración crítica e incluso dramática de la Unión Europea. Presenta cinco grandes prioridades para su acción exterior. En la cuarta: órdenes regionales cooperativos, la Estrategia Global formula una firme y elocuente fundamentación de una gobernanza global de base regional en la que la Unión Europea juega y debe seguir jugando un baza clave.

8. Creciente necesidad y demanda de que la Unión Europea llegue a ser también un actor diplomático.

9. Una pluralidad de actores públicos y privados de diversos niveles interactúan y cooperan sin un Gobierno común en la gobernanza global. De aquí la relevancia de las percepciones entre ellos. Se señalan dos brechas significativas al respecto: la primera, entre cómo la UE se percibe a sí misma y cómo es percibida por los países emergentes; la segunda, entre el reconocimiento del potencial para el liderazgo de la Unión Europea y su liderazgo real. 


\section{Derechos de autor}

Los derechos de autor (para la distribución, comunicación pública, reproducción e inclusión en bases de datos de indexación y repositorios institucionales) de esta publicación (Cuadernos Europeos de Deusto, CED) pertenecen a la editorial Universidad de Deusto. El acceso al contenido digital de cualquier número de Cuadernos Europeos de Deusto es gratuito, transcurridos 6 meses desde su publicación. Los trabajos podrán descargarse, copiar y difundir en cualquier medio sin fines comerciales y según lo previsto por la ley; sin la previa autorización de la Editorial (Universidad de Deusto) o el autor. Así mismo, los trabajos editados en CED pueden ser publicados con posterioridad en otros medios o revistas, siempre que el autor indique con claridad y en la primera nota a pie de página que el trabajo se publicó por primera vez en $C E D$, con indicación del número, año, páginas y DOI (si procede). Cualquier otro uso de su contenido en cualquier medio o formato, ahora conocido o desarrollado en el futuro, requiere el permiso previo por escrito del titular de los derechos de autor.

\section{Copyright}

Copyright (for distribution, public communication, reproduction and inclusion in indexation databases and institutional repositories) of this publication (Cuadernos Europeos de Deusto, CED) belongs to the publisher University of Deusto. Access to the digital content of any Issue of Cuadernos Europeos de Deusto is free only six months after its publication. The content can be accessed, downloaded, copies, and distributed freely in any medium only for non-commercial purposes and in accordance with any applicable copyright legislation, without prior permission from the copyright holder (University of Deusto) or the author. Thus, the content of $C E D$ can be subsequently published in other media or journals, as long as the author clearly indicates in the first footnote that the work was published in $C E D$ for the first time, indicating the Issue number, year, pages, and DOI (if applicable). Any other use of its content in any medium or format, now known or developed in the future, requires prior written permission of the copyright holder. 